\title{
Numerical Simulation of the Flow Field and Pollutant Dispersion around a Hill under Different Thermal Stratifications
}

\author{
Dong-Peng Guo, Peng Zhao, and Ran Wan \\ Department of Environment Science and Engineering, Taiyuan University of Science and Technology, Taiyuan, China \\ Ren-TAi YaO AND Ji-Min Hu \\ China Institute for Radiation Protection, Taiyuan, China
}

(Manuscript received 29 November 2018, in final form 15 September 2019)

\begin{abstract}
This paper applied a commercial computational fluid dynamics code, STAR-CD, with the renormalization group $k-\varepsilon$ turbulence model to simulate the flow and dispersion of contaminants released from a source on the windward side of a hill under different thermal stratifications. In the wake region, the influence of atmospheric stratification on the flow field is inconspicuous under neutral and unstable conditions because of the effect of mechanical disturbance. However, this influence becomes slightly conspicuous under stable conditions. When atmospheric stratification is stable, in the range of $z / H<1.0$ (where $z$ is height above the surface and $H$ is height of the hill), the velocity deficits are smaller than those under neutral and unstable conditions. The maximum turbulence kinetic energy (TKE) appears in the wake regions, and the variation in TKE is significantly lower than that under neutral and unstable conditions. When atmospheric stratification is unstable, the vertical and horizontal spread of the plume is slightly greater than that under neutral and stable conditions and the maximum concentration is less than that under neutral conditions. When the Froude number is large $(\sim 11$; Brunt-Väisälä frequency $=0.52)$, atmospheric stratification is slightly stable, the structure of flow around the hill is generally similar to that under neutral conditions, and the high-concentration regions are large on the windward side of the hill. Smaller high-concentration regions just appear on the windward side of the hill under unstable conditions. The pollutant concentrations in the wake region of the hill increase as a result of the effect of thermal stability, and the vertical spreading range of the plume along the downwind axis ( $x$ axis) is larger than that under neutral and stable conditions.
\end{abstract}

\section{Introduction}

The atmospheric dispersion of radioactive contaminants has always attracted wide attention because it can cause a detrimental effect on the environment in the short term. The dispersion of radionuclide is dependent on meteorological factors such as wind speed and atmospheric stability; in addition, the local concentration change caused by underlying surface conditions, including the terrain, buildings, and ground surface roughness, needs to be considered. The profile of wind velocity and turbulence intensity in the atmospheric boundary layer are influenced by thermal stratification; therefore, thermal stratification dominates pollutant dispersion in the real atmosphere. At present, most dispersion models for

Corresponding author: Guo Dong-Peng, guodp@126.com radioactive contaminants cannot accurately describe the turbulence process resulting from the interaction of wind and obstacles in the 2-km range of the accidental source term, but computational fluid dynamics (CFD) techniques are able to compensate for this limitation. The modeling of unstable and stable atmospheric boundary layers is complicated compared with that for neutral boundary layers. Experimental studies on modeling stable boundary layers remain limited, and these studies concentrated on atmospheric flow under slightly stable conditions [bulk Richardson number $\mathrm{Ri}_{b}<0.25$, where

$$
\mathrm{Ri}_{b}=\frac{g z^{2}\left(d T / d z+\Gamma_{\mathrm{ad}}\right)}{T_{r} u^{2}} \approx \frac{g z_{r} \Delta T}{T_{r} u^{2}},
$$

with $g$ being the gravitational acceleration $\left(9.8 \mathrm{~m} \mathrm{~s}^{-2}\right)$, $T_{r}$ the temperature at the reference height $z_{r}, \Delta T$ 
the temperature difference between the reference height and surface, and $\Gamma_{\mathrm{ad}}$ the dry adiabatic lapse rate]. Yassin et al. (2005) studied the patterns of pollutant dispersion in street canyons under different atmospheric stabilities by performing wind-tunnel experiments and field tests. Yassin (2013) performed a wind-tunnel test to study the effect of thermal stability on the dispersion of rooftop stack emissions around an isolated cubic building. Kanda and Yamao (2016) conducted wind-tunnel tests to study the effect of roughness and atmospheric stability on pollutant dispersion in street canyons. In addition, Takahashi et al. (2005) studied the effect of atmospheric stability on the flow field around a hill under different conditions via a windtunnel test. Hunt and Snyder (1980) studied the flow structure around a three-dimensional hill for Froude number $\mathrm{Fr}=0.1-0.7$ and $\mathrm{Fr}=\infty$ through the towingtank and wind-tunnel tests. The $\mathrm{Fr}=u_{r} /\left(N z_{r}\right)$, where $N$ is the Brunt-Väisälä frequency:

$$
N=\left(\frac{g}{T_{s}} \frac{\partial T}{\partial z}\right)^{1 / 2}
$$

$u_{r}$ is the wind velocity at the reference height, and $T_{s}$ is the surface temperature. The results showed that the spread pattern of plumes from upwind emissions near the hill is dependent on Fr. When Fr is not much greater than 1 , the flow structure round the hill becomes broadly similar to that of a neutral flow; when $\mathrm{Fr}=10$, the stratification still has a strong effect on flow at a distance far downwind. Carruthers and Hunt (1990) studied the flow and turbulence structure over the hills and complex terrain under neutral and slightly stable stratifications $(\mathrm{Fr}>1)$ based on the linear three-dimensional theory and analyzed the influence of a hill on pollutant dispersion. Leo et al. (2016) proposed a calculation method for the dividing-streamline height $H_{L}$ that is based on the Sheppard formula $\left[H_{L} / H=(1-\mathrm{Fr})\right.$, where $H$ is the height of the hill] and a modified logarithmic velocity profile, and they conducted field experiments to investigate the dividing-streamline concept. The results showed that no dividing streamline is discerned for $\mathrm{Fr} \approx 10$, and the predictions from the new formula compare well to experimental observations for $\mathrm{Fr} \approx 0.3-0.4$.

With improvements in computational capability, CFD simulations have been a main approach to study pollutant dispersion under different atmospheric stratifications. Kikumoto et al. (2009) studied the effects of different atmospheric stabilities on the mean wind velocity and pollutant dispersion in a street canyon based on a large-eddy simulation (LES). The results showed that when atmospheric stability is unstable
$\left(\mathrm{Ri}_{b}=-0.21\right)$, the intense motion of airflow produces a stronger turbulence structure in a vertical direction. Thus, unstable stratification results in rapid spreading of pollutants. When atmospheric stability is stable $\left(\mathrm{Ri}_{b}=0.43\right)$, considerable pollutants stagnate in the bottom of canyons because the vertical motion of airflow is weak. Okaze and Mochida (2017) investigated the TKE, temperature, and flow field around an isolated building under unstable conditions $\left(\mathrm{Ri}_{b}=-0.10\right)$ using the LES technique. The results of the LES were in agreement with the experimental data. Olvera and Choudhuri (2006) used the standard $k-\varepsilon$ turbulence model (where $k$ is the turbulent kinetic energy and $\varepsilon$ is the rate of dissipation of $k$ ) to simulate the dispersion of hydrogen and methane around an isolated cubic building under neutral and stable conditions and compared the CFD results with tank-experiment data. The results showed that the influence of the building on the hydrogen dispersion under stable conditions was greater than that under neutral conditions; furthermore, the influence was more significant when the emission source was in the downwind cavity zone. Santos et al. (2009) evaluated the effect of atmospheric stratification on the flow field and pollutant dispersion around an isolated building using a modified $k-\varepsilon$ turbulence model and validated the results using data from a wind-tunnel test and field test. Cheng et al. (2009) used the renormalization group (RNG) $k-\varepsilon$ turbulence model to investigate the influence of streets on the flow and pollutant dispersion. The results indicated that when atmospheric stratification was unstable $\left(\mathrm{Ri}_{b}<0\right)$, a secondary recirculation zone appeared in the upwind direction of the block, increasing the ground-level concentration of pollutants. Furthermore, the recirculation and the ground-level concentrations increased with a decrease in $\mathrm{Ri}_{b}$. Tan et al. (2015) investigated the effects of different thermal stratifications on pollutant dispersion in an urban street. The results indicated that when atmospheric stability is unstable, the inhomogeneous temperature profile significantly changed the structure of the flow field and the dispersion patterns of pollutants in the street. Michalcová et al. (2016) used the shear stress transfer $k-\varepsilon$ turbulence model to simulate thermal stratifications and compared them with wind-tunnel test data. Pontiggia et al. (2009) used the standard $k-\varepsilon$ turbulence model to study the effect of atmospheric stability on pollutant dispersion and conducted a validation based on a windtunnel test. In addition, Tominaga and Stathopoulos (2017) studied the influence of an isolated cubical building on pollutant dispersion using the steady and 
unsteady Reynolds-averaged Navier-Stokes (RANS) equations and validated the simulation results via a wind-tunnel test. Tominaga and Stathopoulos (2018) used different turbulence models to study the patterns of pollutant dispersion around a single cubical building under different thermal stratifications and validated the results using a wind-tunnel test. Furthermore, Chang et al. (2018) studied the effect of thermal stratification on the flow field around a hill using the standard $k-\varepsilon$ turbulence model. Van der Laan et al. (2016) studied the balance between turbulence kinetic energy and dissipation under different thermal stratifications and proposed an equilibrium $k-\varepsilon$ turbulence model based on Monin-Obukhov similarity theory. Pontiggia et al. (2009) achieved the simulation of different atmospheric stratifications by adding an additional source term in the $k-\varepsilon$ equations based on Monin-Obukhov similarity theory and validated the simulated results of neutral and stable conditions using the field test data.

In this paper, the RNG $k-\varepsilon$ turbulence model was used to simulate the dispersion of pollutants released from the upwind source of a three-dimensional hill under different stability conditions. The influence of thermal stratifications on the flow and concentration distribution was examined in the vicinity of the hill. The three-dimensional hill model used in the simulation was the same as that in the wind-tunnel test performed by Takahashi et al. (2005). The test section was $16.5 \mathrm{~m}$ long, $2.2 \mathrm{~m}$ wide, and $1.8 \mathrm{~m}$ high in the wind tunnel. The model was constructed on a geometric scale of 1:1000. The height $H$ and base diameter $D$ of the three-dimensional hill model of cosine type were 200 and $840 \mathrm{~mm}$, respectively. The shape of the model was described using the following relationship:

$$
H_{i}=0.5 H\left[1+\cos \left(2 \pi x_{i} / D\right)\right],
$$

where $x_{i}$ is a horizontal distance, $H_{i}$ is the height at the given distance $x_{i}$, and the center of the base of the model is at the origin of the coordinate. The maximum slope of the hill is $25.5^{\circ}$.

\section{Numerical simulation}

The commercial CFD code STAR-CD 3.26 (developed by $\mathrm{CD}$-adapco) was used to perform the simulation. To compare the results of the wind-tunnel test, a computational domain of $16.5 \mathrm{~m} \times 2.2 \mathrm{~m} \times 1.8 \mathrm{~m}$ (length $L_{d} \times$ width $W_{d} \times$ height $H_{d}$ ) was used. A three-dimensional hill model of cosine type was adopted, which was identical to that reported by Takahashi et al. (2005). To study the effect of the hill on the dispersion of pollutants from upwind emission, a stack was set at the upwind point $x / H=-2.5$. The height $H_{s}$ and inner diameter of the stack were $100(0.5 H)$ and $4 \mathrm{~mm}$, respectively. A schematic of the stack and hill in the domain is shown in Fig. 1. Ethylene $\left(\mathrm{C}_{2} \mathrm{H}_{4}\right)$, a passive scalar, was used as the tracer, and its temperature was the same as the ambient temperature. The equation $K=C^{*} U_{t} H^{2} / Q$ was used to calculate the normalized concentrations, where $C^{*}$ is the volume concentration, $U_{t}$ is the mean stream velocity at the top of the simulated boundary layer, and $Q$ is the volume emission rate of gas (the exit velocity is one-half of the free stream velocity at the corresponding height). The computational domain was discretized in hexahedral cells, which have a good topological structure. The computational mesh around the hill is shown in Fig. 2. The total number of cells was approximately 3 million. The maximum grid size was $30 \mathrm{~mm}$, and the minimum grid size was $5 \mathrm{~mm}$. The grid-independent test was performed using two additional meshes, and the minimum grid sizes are $2 \mathrm{~mm}$ (fine mesh) and $10 \mathrm{~mm}$ (coarse mesh), respectively. As shown in Fig. 3, the velocity differences between the different meshes are very small. In comparison with the finest meshes, the relative errors $\left(100 \% \times\left|U-U_{\text {fine }}\right| / U_{\text {fine }}\right)$ from the default and coarse meshes are broadly small. The errors using default meshes are relatively smaller than those using coarse meshes. The maximum error is less than $5 \%$ and appears near the ground. The errors are less than $1 \%$ above the height of $0.07 \mathrm{~m}$. Considering computation cost and result accuracy, the 5-mm grid was used in the simulation.

The equilibration between the turbulence model and boundary conditions is necessary in a RANS model. The CFD simulations should be examined the equilibration in the simulated boundary layer. The retention of the simulated boundary layer was verified using an empty domain simulation. The vertical profile of velocity satisfies a power law with an exponent of 0.143 . The vertical profiles of velocity at three central-axis positions are compared with the approaching flow, and the differences are negligible. The downstream developments of the approaching flow are shown in Fig. 4.

\section{a. Turbulence model}

The Navier-Stokes equations are able to describe the flow in the atmosphere. Air can be regarded as an incompressible fluid because its flow velocity is generally small. Turbulence is the main form of atmospheric motion in the surface layer. At present, governing equations are solved based on the Reynolds-average method. The RANS equations introduce the second-order fluctuation correlation quantity. The turbulence model is the 


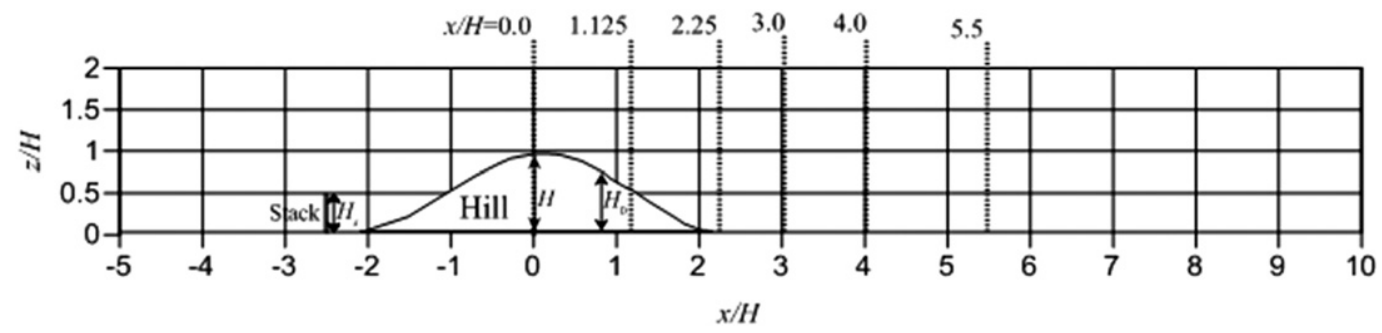

FIG. 1. A schematic of the stack and hill in the computational domain.

specific correlation that combines the additional term due to turbulence with the time-average values. The temperature variation of ambient air is very small, and the density of air can be regarded as a constant. Therefore, airflow satisfies the Boussinesq assumption. The buoyancy can be represented as a function of temperature difference, and a buoyancy source term $\left[S_{B}=\rho \beta\left(T_{\mathrm{amb}}-T\right) g\right.$, where $\rho$ is the density, $T_{\mathrm{amb}}$ is the ambient temperature, and $\beta=1 / T$ is the expansion coefficient] is added in the momentum equation. Calculating the turbulence viscosity is key to solving the equations. Various turbulence models are defined according to different solving methods, such as the standard $k-\varepsilon$ turbulence model, which cannot accurately describe the complicated flow characteristic of the reverse flow region (Murakami 1993). However, the RNG $k-\varepsilon$ turbulence model is better at addressing reverse flow problems (Tominaga et al. 2008). This paper solves the closure Navier-Stokes equations, combining the RNG $k-\varepsilon$ turbulence model (Yakhot et al 1992). The equations of the turbulence model are described in Eqs. (1). The energy equilibrium equation was able to consider the effect resulting from temperature change. To simulate different atmospheric stratifications, the turbulence model was modified by adding an additional source term $S_{\varepsilon}$ as proposed by Pontiggia et al. (2009). The turbulence model constants are listed in Table 1.

$$
\begin{aligned}
& \frac{\partial}{\partial t}(k)+\frac{\partial}{\partial x_{j}}\left[k u_{j}-\left(\mu+\frac{\mu_{t}}{\sigma_{k}}\right) \frac{\partial k}{\partial x_{j}}\right] \\
&=\mu_{t}\left(P_{T}+P_{B}\right)-\varepsilon-\frac{2}{3}\left(\mu_{t} \frac{\partial \mu_{i}}{\partial x_{i}}+k\right) \frac{\partial u_{i}}{\partial x_{i}}
\end{aligned}
$$

$$
\begin{aligned}
\frac{\partial}{\partial t}(\varepsilon)+\frac{\partial}{\partial x_{j}}\left[\varepsilon u_{j}-\left(\mu+\frac{\mu_{t}}{\sigma_{\varepsilon}}\right) \frac{\partial \varepsilon}{\partial x_{j}}\right]= & C_{\varepsilon 1} \frac{\varepsilon}{k}\left[\mu_{t} P_{T}-\frac{2}{3}\left(\mu_{t} \frac{\partial \mu_{i}}{\partial x_{i}}+k\right) \frac{\partial \mu_{i}}{\partial x_{i}}\right]+C_{\varepsilon 3} \frac{\varepsilon}{k} \mu_{t} P_{B} \\
& -C_{\varepsilon 2} \frac{\varepsilon^{2}}{k}+C_{\varepsilon 4} \varepsilon \frac{\partial u_{i}}{\partial x_{i}}-\frac{C_{\mu} \eta^{3}\left[1-\left(\eta / \eta_{0}\right)\right]}{1+\beta \eta^{3}} \frac{\varepsilon^{2}}{k}+S_{\varepsilon}(z),
\end{aligned}
$$

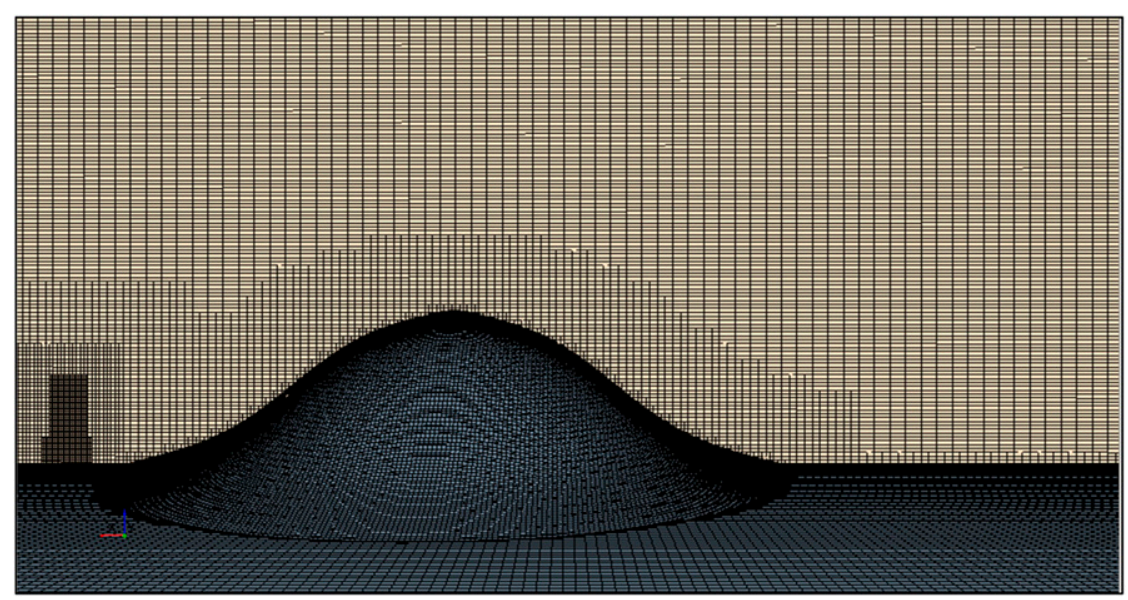

FIG. 2. Computational mesh in the domain. 
(a)

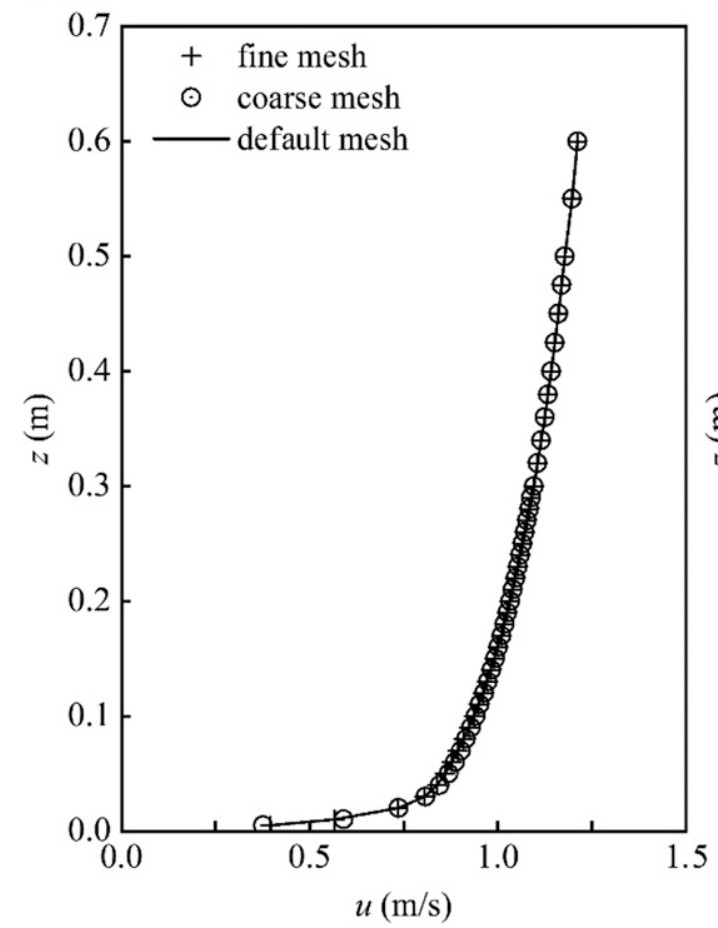

(b)

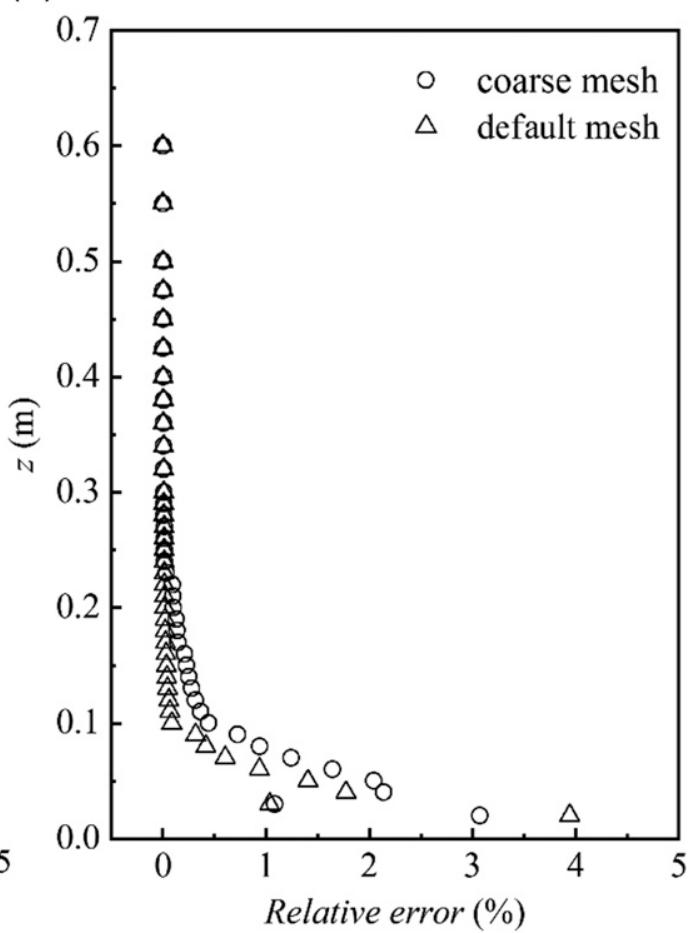

FIG. 3. Grid-independent test: (a) vertical profiles of the $u$ velocity, and (b) relative errors of the fine and coarse meshes.

with

$$
\begin{aligned}
P_{T} & =S_{i j} \frac{\partial u_{i}}{\partial x_{i}}, \\
P_{B} & =-\frac{g_{i}}{\sigma_{h}} \frac{\partial T}{\partial x_{i}}, \\
\eta & =S \frac{k}{\varepsilon}, \\
S_{i j} & =\frac{\partial u_{i}}{\partial x_{j}}+\frac{\partial u_{j}}{\partial x_{i}}, \\
S & =\frac{k}{\varepsilon}\left(0.5 S_{i j} S_{i j}\right)^{1 / 2}, \quad \text { and } \\
\frac{\partial T}{\partial t}+\nabla \cdot\left(v c_{p} T\right) & =\nabla \cdot\left(k_{T} \nabla T\right),
\end{aligned}
$$

where $k$ is the TKE; $\varepsilon$ is the turbulence dissipation rate; $\mu$ is the viscosity; $\mu_{t}$ is the turbulence viscosity; $S_{i j}$ is the mean velocity strain rate; $P_{T}$ is the turbulence stress; $P_{B}$ is the production of TKE by buoyancy forces; $\sigma_{k}, \sigma_{\varepsilon}$, and $\sigma_{h}$ are the turbulent Prandtl numbers; $C_{\varepsilon 1}, C_{\varepsilon 2}, C_{\varepsilon 3}$, and $C_{\mu}$ are empirical constants; $\eta_{0}$ and $\beta$ are empirical coefficients; $S_{\varepsilon}$ is the source term; $T$ is the temperature at the different heights $K ; k_{T}$ is the thermal conductivity; and $c_{p}$ is the specific heats $\left(\mathrm{J} \mathrm{kg}^{-1} \cdot \mathrm{K}^{-1}\right)$.

\section{b. The source term $\left(S_{\varepsilon}\right)$}

This paper used the Monin-Obukhov length $L$ to describe the atmosphere turbulence motion under different atmospheric stratifications. The $L$ is an estimation of the height where the turbulent dissipation due to the buoyancy is comparable with the shear stress production of turbulence and it can be expressed by the following relation (Pontiggia et al. 2009):

$$
L=\frac{u_{*}^{2} T_{w}}{\kappa g T_{*}},
$$

with

$$
\begin{aligned}
& u_{*}=\left(\tau_{w} / \rho\right)^{1 / 2} \text { and } \\
& T_{*}=\frac{-q_{w}}{\rho c_{p} u_{*}}
\end{aligned}
$$

where $u_{*}$ is the friction velocity, $T_{*}$ is the surface-layer temperature scale, $q_{w}$ is the surface heat flux $\left(\mathrm{W} \mathrm{m}^{-2}\right)$, and $\tau_{w}$ is the surface shear stress.

The $q_{w}$ and the bulk Richardson number are equal to zero under neutral conditions, and the $q_{w}$ and $T_{*}$ for the nonneutral conditions are calculated on the basis of Monin-Obukhov similarity theory. The $L$ is infinite and the $T_{*}$ tends to zero under neutral conditions. 


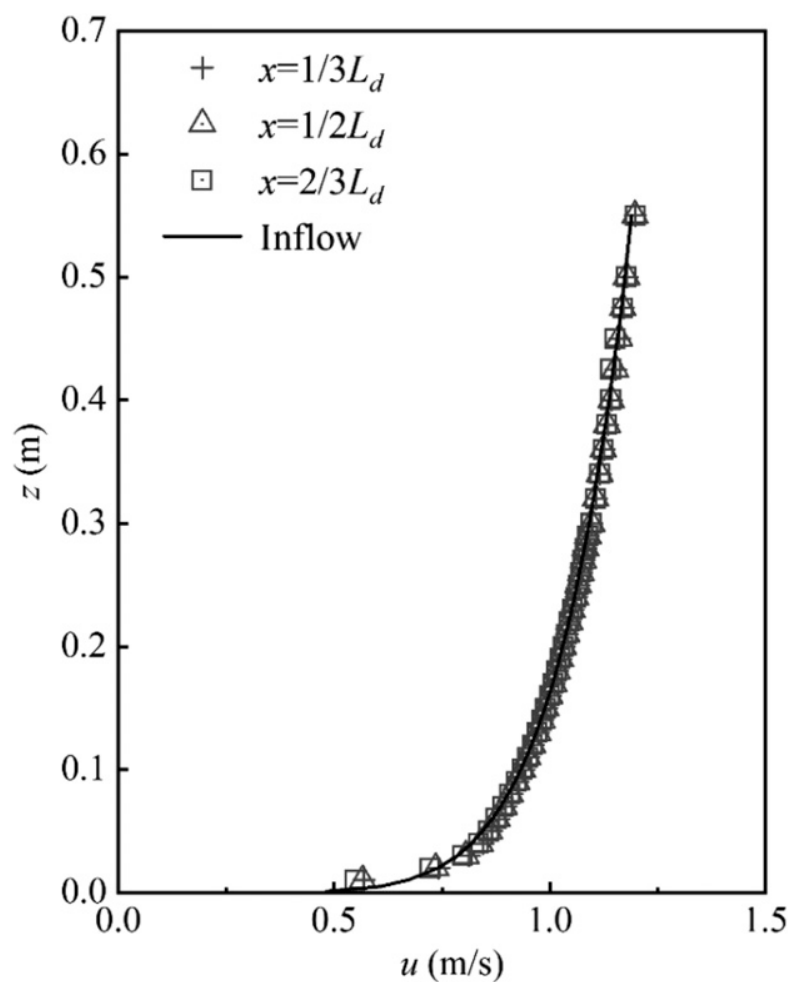

FIG. 4. The profiles of inflow velocity at different positions in the empty domain.

Hence, the source term $S_{\varepsilon}(z)$ for neutral conditions is described by Eq. (11):

$$
S_{\varepsilon}(z)=\frac{\rho u_{*}^{4}}{z^{2}}\left[\frac{\left(C_{2 \varepsilon}-C_{1 \varepsilon}\right) \sqrt{C_{\mu}}}{\kappa^{2}}-\frac{1}{\sigma_{\varepsilon}}\right]-\mu_{t} \frac{u_{*}^{3}}{2 \kappa z^{3}} .
$$

The $L$ is a constant and the $T_{*}$ is nonzero under nonneutral conditions. The $S_{\varepsilon}(z)$ for the unstable and stable conditions is described by Eq. (12):

$$
\begin{aligned}
S_{\varepsilon}(z)= & \frac{\rho u_{*}^{4}}{z^{2}}\left[\frac{\left(C_{2 \varepsilon}-C_{1 \varepsilon}\right) \sqrt{C_{\mu}}}{\kappa^{2}} \Phi_{\varepsilon}^{2} \sqrt{\frac{\Phi_{\varepsilon}}{\Phi_{m}}}\right. \\
& \left.-\frac{1}{\sigma_{\varepsilon}}\left(\frac{2}{\Phi_{m}}-\frac{1}{\Phi_{m}^{2}}+\frac{T_{*}}{\kappa T}\right)\right]-\mu_{t} \frac{2 u_{*}^{3}}{\kappa z^{3}},
\end{aligned}
$$

where $\Phi_{m}$ and $\Phi_{\varepsilon}$ are modified functions that depend on $z / L$ (Panofsky and Dutton 1984). The $\Phi_{m}$ tends to 1 under neutral conditions. The values of $z / L$ for different atmospheric stratifications are shown in Table 2. For unstable conditions $(z / L \leq 0)$,

$$
\Phi_{m}=(1-16 z / L)^{-1 / 4} \quad \text { and } \quad \Phi_{\varepsilon}=(1-16 z / L)^{-1 / 2} .
$$

TABLE 1. Turbulence model constants.

\begin{tabular}{cccccccccc}
\hline \hline$C_{\mu}$ & $C_{\varepsilon 1}$ & $C_{\varepsilon 2}$ & $C_{\varepsilon 3}$ & $C_{\varepsilon 4}$ & $\sigma_{k}$ & $\sigma_{\varepsilon}$ & $\sigma_{h}$ & $\eta_{0}$ & $\beta$ \\
\hline 0.085 & 1.42 & 1.68 & 1.42 & -0.387 & 0.719 & 0.719 & 0.9 & 4.38 & 0.012 \\
\hline
\end{tabular}

For stable conditions $(z / L \geq 0)$ :

$$
\Phi_{m}=1+5 z / L \text { and } \Phi_{\varepsilon}=1+4 z / L .
$$

\section{c. Wall functions}

The shear stress turbulence production is particularly important near the ground. The standard wall function is usually used to describe the flow near the wall in the CFD simulation. The methods using the standard wall function are presented below. The formula of Newton shear stress is applicable in the nearest cells of the wall:

$$
\tau_{w}=-\lambda_{w}\left(\overline{U_{p}}-\overline{U_{w}}\right)
$$

where $U_{p}$ and $U_{w}$ are the velocity in the cells and the motion velocity of the wall, $U_{w}=0$, in this simulation. The $\lambda_{w}$ is calculated according to the distance from the wall to the cell center. The calculation formulas are below:

$$
\begin{array}{ll}
\lambda_{w}=\mu / y p, & y_{p}^{+}<11 \quad \text { and } \\
\lambda_{w}=\rho C_{\mu}^{1 / 4} k_{p}^{1 / 2} k / \ln \left(E y_{p}^{+}\right), & y_{p}^{+}>11,
\end{array}
$$

where $y_{p}$ is the vertical distance from the nearest cellcenter to the wall, $y_{p}^{+}=\left(\rho C_{u}^{1 / 4} k_{p}^{1 / 2} y\right) / \mu$ is the nondimensional distance from the wall to cell center, $\kappa=0.4$, and $E=9.0$. The $\tau_{w}$ is the surface shear stress. The calculation value of $\tau_{w}$ can be as an additional source term in the momentum equation of the center of cells near the wall, to replace the turbulence stress on the corresponding governing surface.

\section{d. Boundary conditions}

The vertical profile of wind velocity in the atmospheric boundary layer is mainly dependent on the atmospheric stability and the surface roughness. The wind velocity increases with increasing elevation, namely, the wind gradient. Different simulated boundary layers are achieved by changing the temperature gradients in the vertical direction and setting different temperatures in the top and bottom of the domain. The simulation for atmospheric flow takes into account the influence of thermal stratification. Simulated thermal stratification is mainly dependent on the Reynolds number $\left(\mathrm{Re}=u_{s} H_{s} / \nu, u_{s}\right.$ is the velocity at the stack height $H_{s}$, and $\nu$ is the kinematic viscosity) and the bulk 
TABLE 2. The main parameters of different thermal stratifications.

\begin{tabular}{lccccccccc}
\hline \hline Stability class & $u_{r}\left(\mathrm{~m} \mathrm{~s}^{-1}\right)$ & $u_{H}\left(\mathrm{~m} \mathrm{~s}^{-1}\right)$ & $z_{0}(\mathrm{~m})$ & $\operatorname{Re}$ & $\operatorname{Ri}(H=0.2 \mathrm{~m})$ & $z / L$ & $\mathrm{Fr}$ & $N\left(\mathrm{~s}^{-1}\right)$ & $\left.q_{w}(\mathrm{~W} \mathrm{~m})^{-2}\right)$ \\
\hline Unstable & 0.8 & 1.23 & 0.004 & $1.76 \times 10^{4}$ & -0.002 & -0.002 & - & - & 8.5 \\
Neutral & 0.67 & 1.03 & 0.006 & $1.47 \times 10^{4}$ & 0.0 & 0 & $\infty$ & 0 & 0 \\
Stable & 0.75 & 1.15 & 0.002 & $1.64 \times 10^{4}$ & 0.008 & 0.009 & 11 & 0.52 & -10.4 \\
\hline
\end{tabular}

Richardson number. The Reynolds number should be greater than 11000 at the height of the stack (Snyder 1981). The Reynolds numbers at the height of the stack under different atmospheric stratifications are shown in Table 2.

In the simulation, the profiles of wind velocity, turbulence intensity, and temperature at the inlet boundary were set according to the wind-tunnel test reported by Takahashi et al. (2005). The vertical profile of inlet wind velocity was described by a power-law equation:

$$
u=u_{r}\left(z / z_{r}\right)^{n}
$$

where $u$ is the velocity at the given height $z, u_{r}$ is the wind velocity at the reference height $z_{r}, z_{r}=0.01 \mathrm{~m}$, and the wind shear exponent $n=0.143$. The profiles of wind velocity, turbulence and temperature at the inlet boundary are shown in Fig. 5. The profile of turbulence dissipation in the vertical direction is described by $\varepsilon(z)=\left(u_{*}^{3} / \kappa z\right) \Phi_{\varepsilon}$.

A fully developed flow assumption was used for the outlet boundary. As the two sides and the top of the domain are a long way from the hill and parallel to the wind direction, the velocity gradients along the tangent and normal directions were taken to be zero. Therefore, a slip wall condition was used for these boundaries. A specific friction velocity $u_{*}$ and roughness $z_{0}$ were set for the ground surface, and a no-slip condition was applied to the ground. Flow near the wall was calculated by wall functions. A curved boundary condition was used for the surface of the hill.

The semi-implicit method for pressure linked equations (SIMPLE; Patankar and Spalding 1972) algorithm was used for pressure-velocity coupling. The different scheme was quadratic upwind interpolation of convective kinematics (QUICK). In the calculation, pressure, momentum, TKE, and the turbulence dissipation rate were subjected to relaxation processing.

\section{Results and discussion}

Numerical simulations were performed for three cases (neutral, unstable, and stable atmospheric conditions). To study the effect of different thermal stratifications on the dispersion and flow near the recirculation region on the leeward side of the hill, the profiles of velocity and concentrations at six different positions
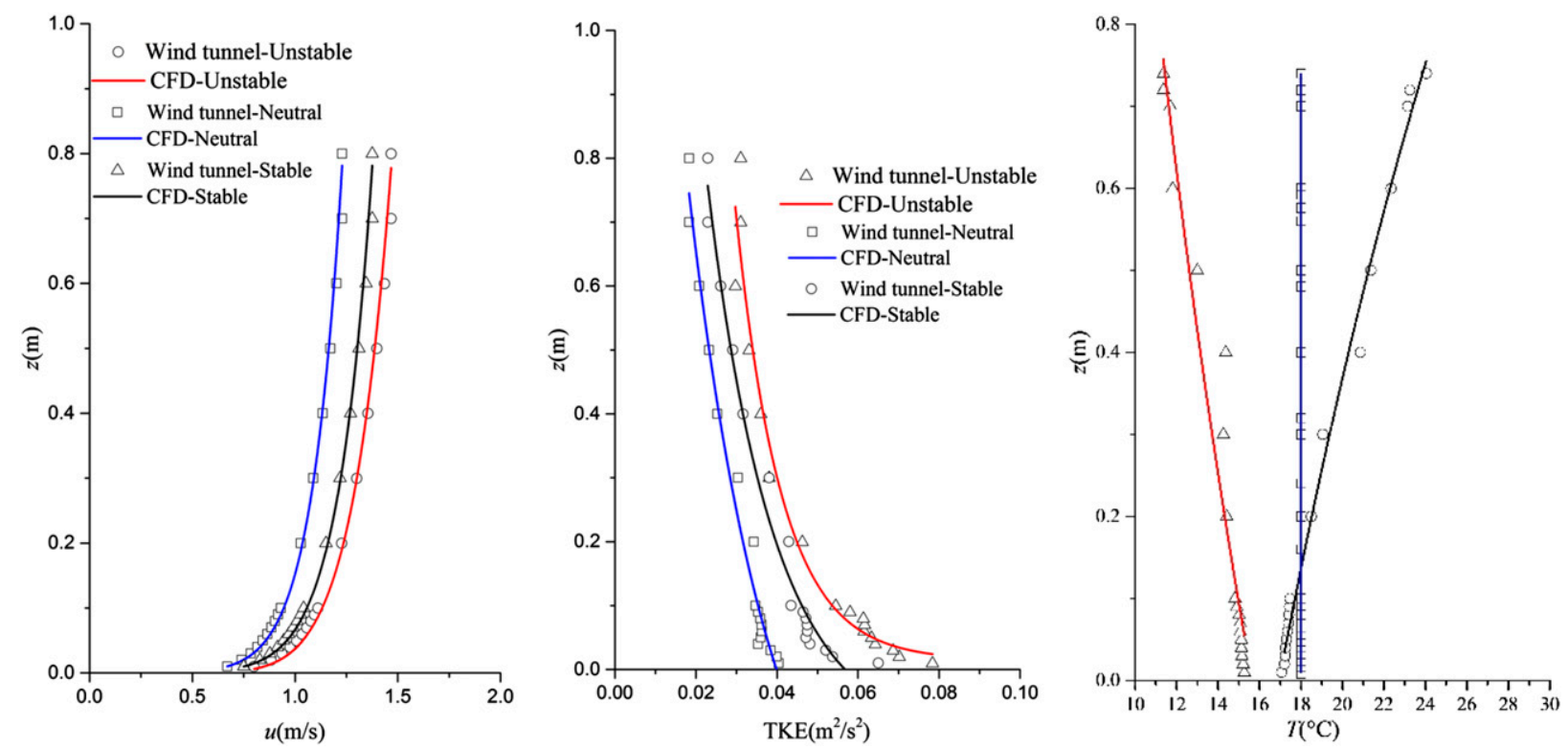

FIG. 5. Vertical distributions of (left) mean velocity, (center) TKE, and (right) temperature in the simulated boundary layer under thermal stability. 
$(x / H=0.0,1.125,2.25,3.0,4.0$, and 5.5; see Fig. 1) along the central axis $(y=0)$ of the hill under different conditions were compared and analyzed.

\section{a. Flow field}

The velocities were normalized to facilitate comparison. The normalized velocity $\left(u / u_{H}\right.$ and $\left.w / u_{H}\right)$ was the ratio of the mean velocity in a specific direction (horizontal $u$ or vertical $w$ ) relative to the mean inflow velocity $u_{H}$ at the top of the hill $(H=200 \mathrm{~mm})$.

\section{1) The HORIZONTAL VELOCITY PROFILE $U / U_{H}$}

Figure 6 compares the horizontal normalized velocities $\left(u / u_{H}\right)$ of the simulation and wind-tunnel test at different positions. The results of the simulation are in approximate agreement with the test data. The greatest variation in wind velocity is observed near the hilltop, and the velocities are generally smaller in the recirculation region on the leeward side of the hill under different stability conditions. When atmospheric stability is stable, the velocity deficit is slightly higher than that under neutral and unstable conditions, and the greatest deficit is approximately $20 \%$ of the inflow velocity at the corresponding height. As the downwind distance increases, the velocity gradually recovers to the inflow state because the influence of the hill on the flow weakens. However, the recovery of velocity is relatively slower under stable conditions. This indicates that, for a typical steep hill, the wake effect nearly disappears at the downwind distance $x / H=5.5$.

As shown in Fig. 6, at the point of the hilltop $(x / H=0)$, the change in wind velocity is dominated by the mechanical disturbance over the hilltop. Therefore, the velocity variation resulting from thermal stratification is very small. At the point $x / H=0, u / u_{H}=1.14$ for the stable condition, $u / u_{H}=1.08$ for the neutral condition and $u / u_{H}=1.13$ for the unstable condition at the lowest measuring height. In the wake region of the hill $(x / H=$ 1.125 and 2.25), the $u / u_{H}$ value is relatively large under unstable and neutral conditions compared to under stable conditions, particularly in the range of $z / H<1.0$. This is mainly because of the mixing characteristics of the stable boundary layer (Takahashi et al. 2005). At the point of $x / H=1.125$, the $u / u_{H}$ value near the ground for the unstable, neutral and stable conditions is $-0.12,-0.24$, and -0.32 , respectively. A reverse flow region is observed on the leeward side of the hill under different conditions, and the recirculation region appears near the height of $z / H=0.75$. The results are in good agreement with the test data. When atmospheric stratification is stable, the velocity deficit in the range of $z / H<1.0$ is less than that under neutral and unstable conditions, particularly at the point of $x / H=3.0$. However, at the point of $x / H=0.0,1.125$, and 2.25 , the effect of thermal stratification on the flow field is inconspicuous under neutral and unstable conditions but slightly conspicuous under stable conditions. This is because the influence of mechanical disturbance produced by a hill is dominant.

\section{2) THE VERTICAL VELOCITY PROFILE $W / U_{H}$}

The comparisons of the vertical normalized velocities $\left(w / u_{H}\right)$ in the simulation and the wind-tunnel test are shown in Fig. 7. The results of the simulation are in approximate agreement with the test data. However, at the point of $x / H=1.125$ and 2.25 , the discrepancy between the simulation results and the test data is relatively large in the range of $z / H>1.75$. As shown in Fig. 7, the vertical velocities at different positions are generally small. At the point of the hilltop $(x / H=0)$, all the vertical velocities at the different heights become negative under different conditions. This is because the direction of airflow is downward over the hilltop. In the range of $z / H>1.0$, the values of $w / u_{H}$ at the different positions are less than 0 . This is potentially because the effect of thermal stratification caused airflow to generate slight downward movement. At the point of $x / H=1.125$ and 2.25 , the magnitude of $w / u_{H}$ in the range of $z / H<1.0$ under the unstable conditions is greater than that under the neutral conditions, and that under the stable conditions is lower than that under the neutral conditions. The influence of atmospheric stability on the $w / u_{H}$ value can be neglected in the range of $x / H>3.0$.

\section{3) STRUCTURE OF THE FLOW FIELD}

The flow field on the center plane of the hill under different conditions is shown in Fig. 8. The numerical simulation is able to describe the details of the wake region and the recirculation region at the lee of the hill. There is a significant change in the flow field on the windward side of the hill. The wind velocity near the windward side translates into a vertical component and a horizontal component. Reverse flows appear in the region near the leeward side of the hill, and the local velocities become straight downward. The effects of thermal stratifications on the flow field are similar under different conditions. The height of cavity zone is about $0.5 H$ for the unstable condition, $0.75 \mathrm{H}$ for the neutral condition, and $0.8 \mathrm{H}$ for the stable condition. The attachment points behind the hill under neutral, stable, and unstable conditions are, respectively, $1.9 \mathrm{H}, 2.9 \mathrm{H}$, and $2.4 \mathrm{H}$. The height and length of cavity zone under stable conditions are slightly larger than those under neutral and unstable conditions. 


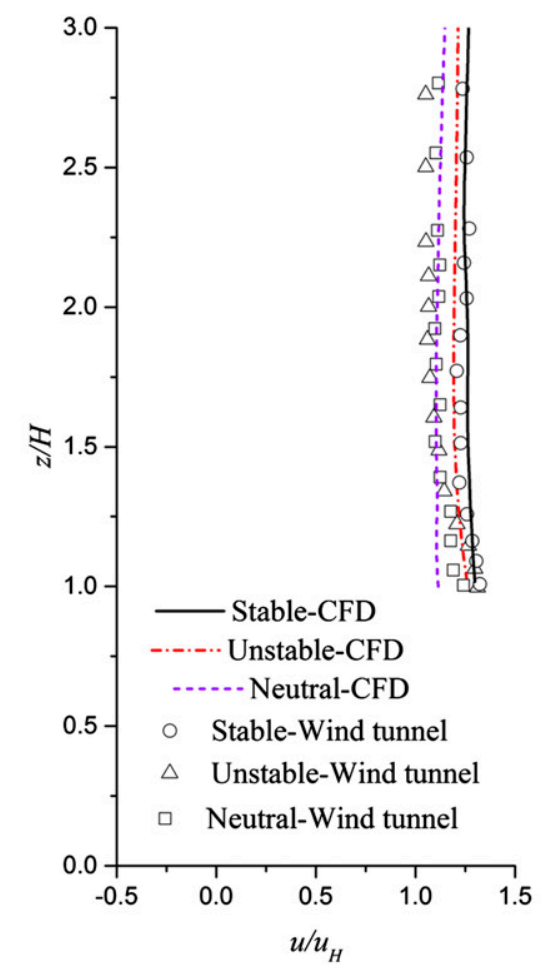

$x / H=0.0$

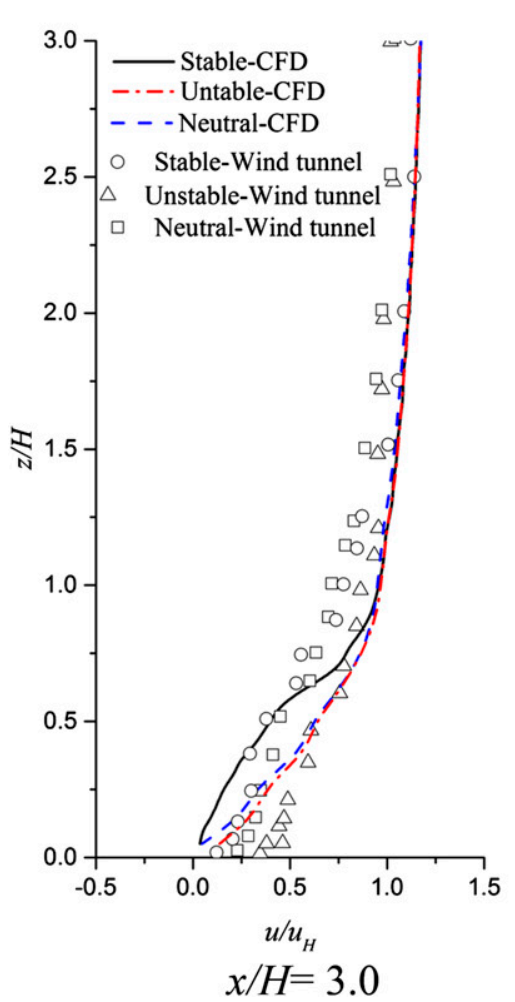

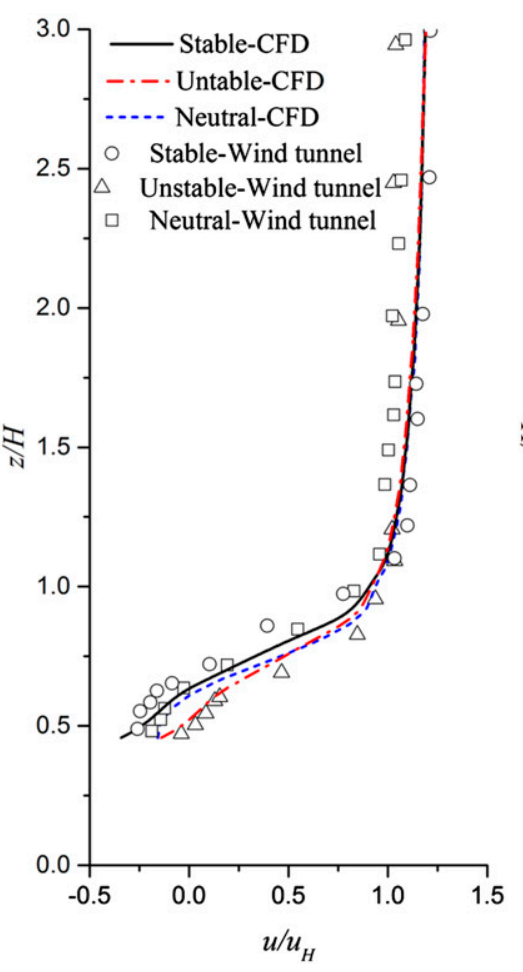

$x / H=1.125$

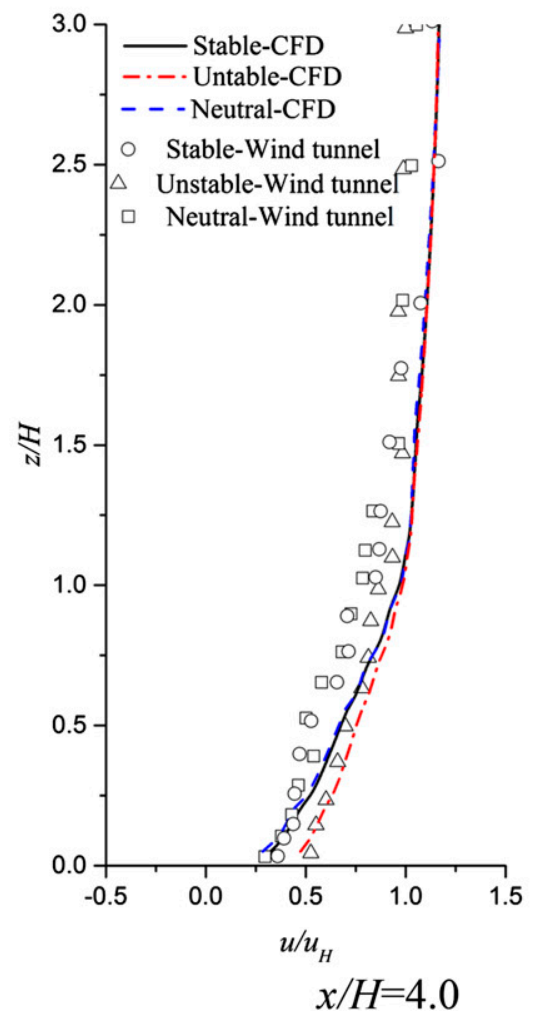

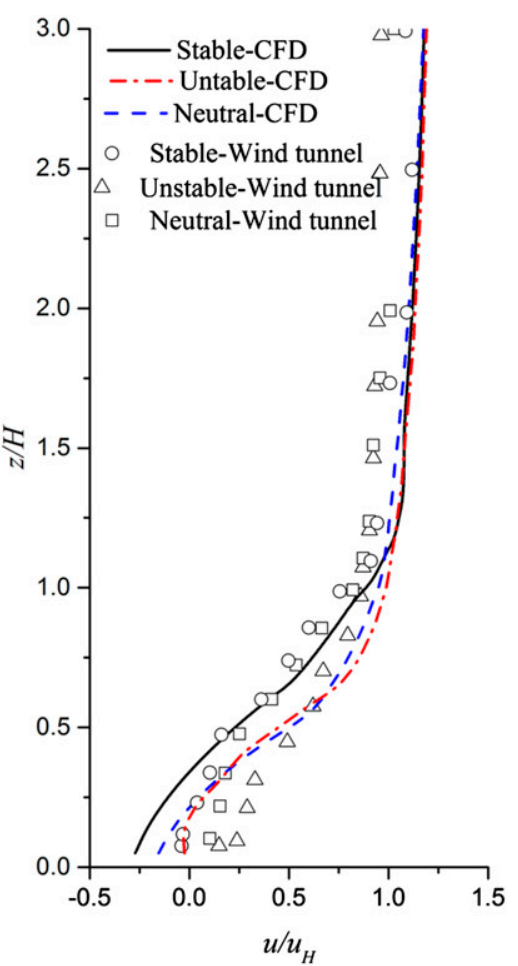

$x / H=2.25$

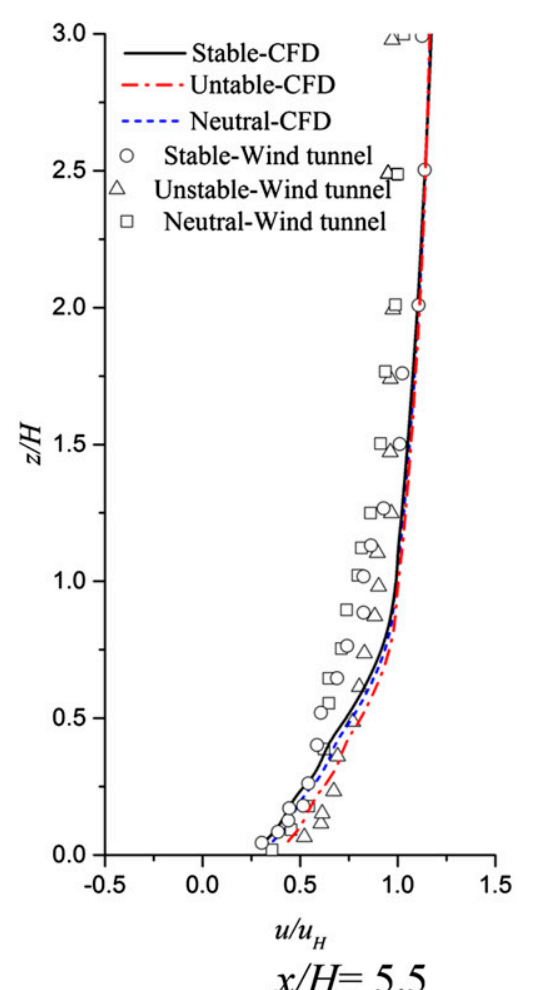

FIG. 6. Vertical profiles of mean velocity component in the longitudinal direction, $u / u_{H}$. 

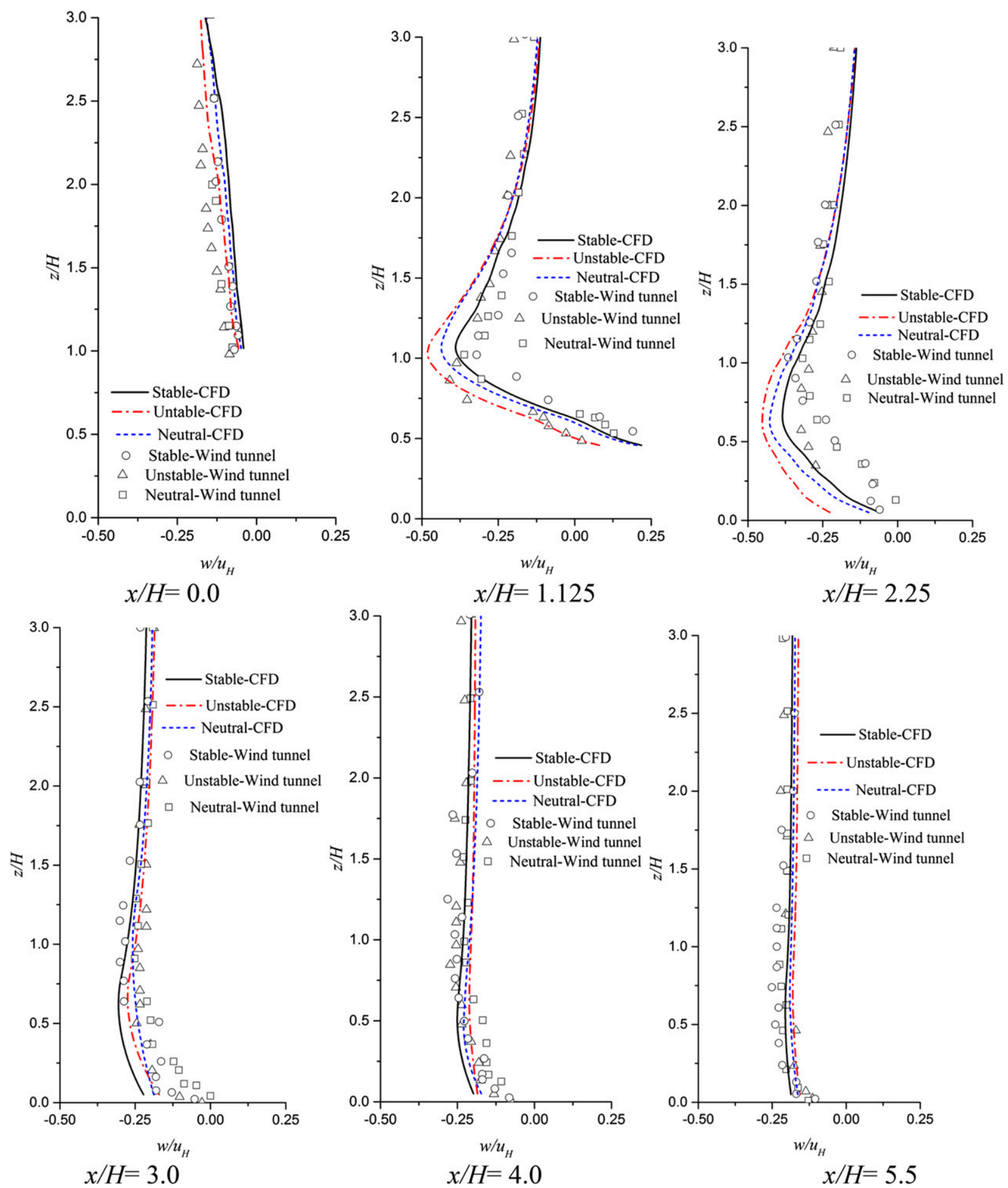

$x / H=2.25$ $x / H=3.0$

$$
x / H=4.0
$$

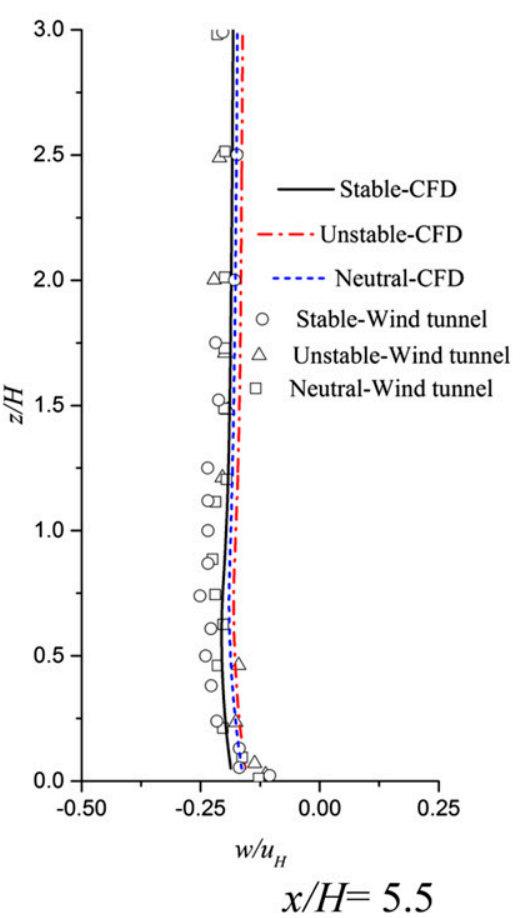

FIG. 7. Vertical profiles of mean velocity component in the vertical direction, $w / u_{H}$.

There is not conspicuous disturbance and deflection in the upwind direction of the hill. This is because when $\mathrm{Fr}$ is relatively larger $(\mathrm{Fr} \approx 11 ; N=0.52)$, the structure of flow around the hill under slightly stable conditions is generally similar to that under neutral conditions. Hunt and Snyder (1980) conducted the towing tank and windtunnel test and found that when Fr is not much greater than 1 , the flow structure round the hill becomes broadly 


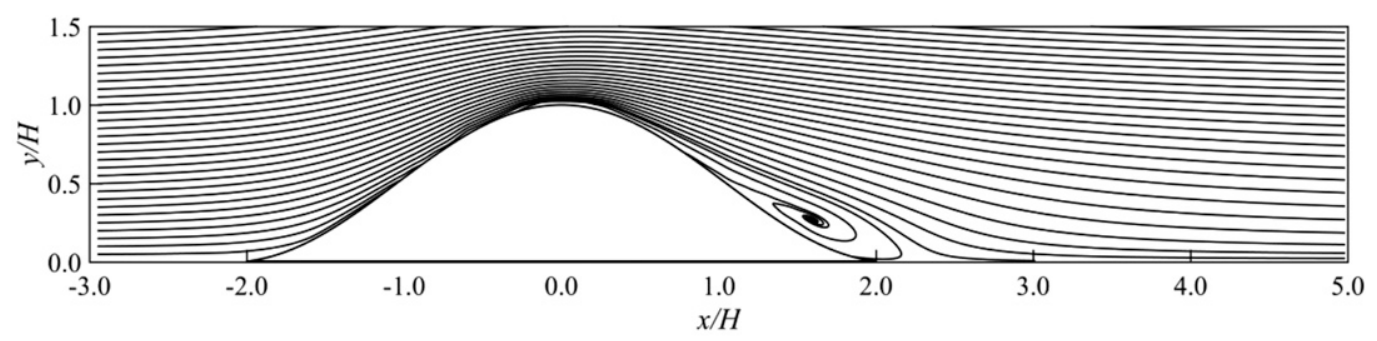

(a) Unstable conditions

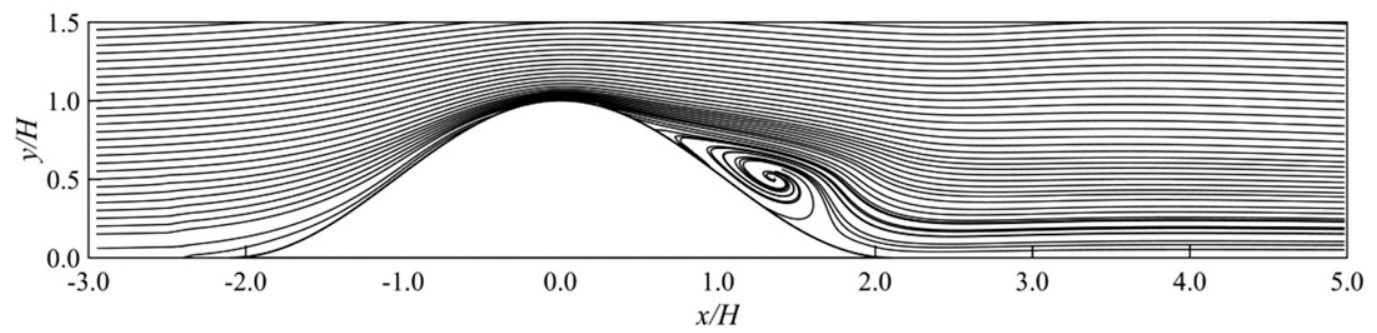

(b) Neutral conditions

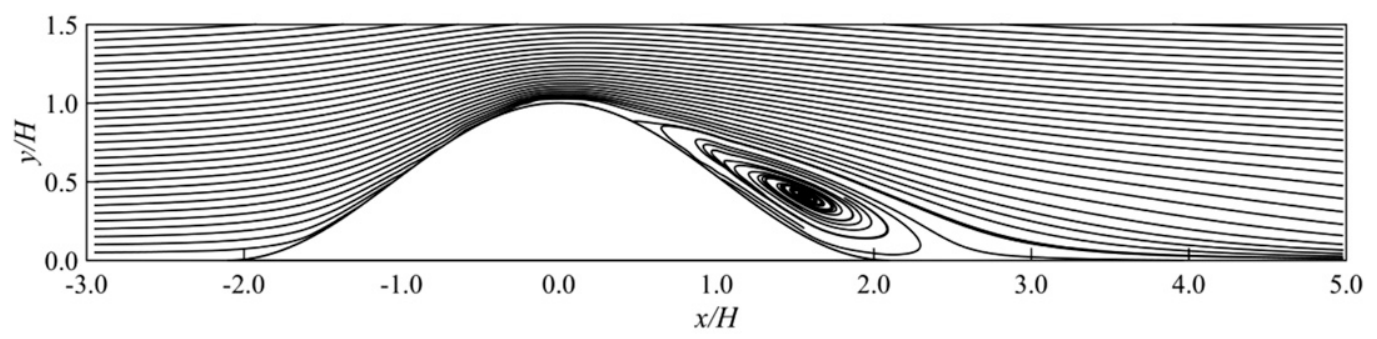

(c) Stable conditions

FIG. 8. The flow field on the center plane of the hill under different conditions.

similar to that of neutral flow; for $\mathrm{Fr} \approx 10$, the stable stratification still has a strong effect on flow at a far downwind distance. Leo et al. (2016) conducted field experiments to study the dividing-streamline concept and found that when $\mathrm{Fr} \approx 10$, it is too large to generate a dividing streamline in the midheights of the hill. The results in this work are consistent with the research reported by Hunt and Snyder (1980) and Leo et al. (2016).

Finnigan (1988) analyzed the experimental data from the field and wind-tunnel test and found that when the maximum slope is less than $0.27\left(15^{\circ}\right)$, a reverse flow region does not appear on the leeside of the hill; when the maximum slope is larger than $0.32\left(18^{\circ}\right)$, a reverse flow region is always observed on the leeward side of the hill; when the maximum slope is in the range of 0.27 and 0.32 , the appearance of reverse flow region is uncertain. However, Wood (1995) indicated the critical slope of separate flow is $0.63\left(32^{\circ}\right)$ for a three-dimensional hill.

In this paper, the maximum slope of the hill is $25.5^{\circ}$ and reverse flow is observed on the leeward side of the hill under different atmospheric stratifications. The results of the work are consistent with the study reported by Finnigan (1988).

\section{b. The turbulence profile $k / u_{H}^{2}$}

Figure 9 compares the profiles of normalized TKE $\left(k / u_{H}^{2}\right)$ in the simulation and wind-tunnel test. The results of the numerical simulation are in approximate agreement with the test data. The maximum $k / u_{H}^{2}$ value appears in the wake region of the hill. This is because the mechanical disturbance caused by the hill produces large velocity gradients. The separation of airflow increases the TKE at the hilltop, and the disturbance tendency continuously extends downstream of the hill. When atmospheric stratification is stable, the change in $k / u_{H}^{2}$ is slightly smaller than that of unstable and neutral conditions. Stable stratification suppresses the convective motion of the atmosphere in the vertical direction, which decreases the variation of TKE. Furthermore, TKE significantly influences the flow field and concentration 

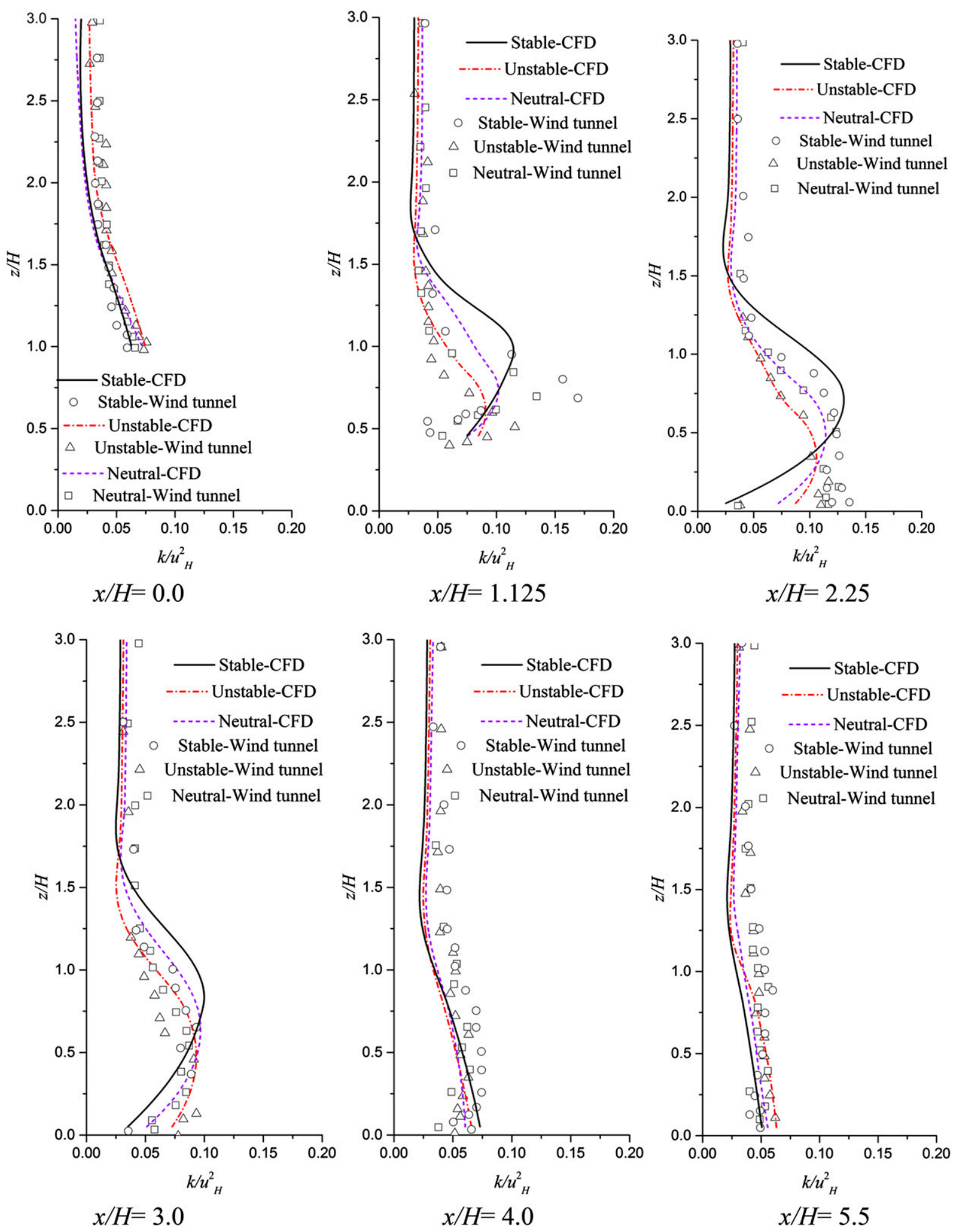

FIG. 9. The profiles of normalized TKE, $k / u_{H}^{2}$. 
distribution. As shown in Fig. 9, in the range of $z / H<1.5$, the discrepancy between the simulated results and windtunnel experiment data is relatively larger in the reverse flow region $(x / H=1.125,2.25$, and 3.0). This is because the RANS model is not able to properly address the separation of flow and overestimates the size and strength of the revers flow region (Meroney et al. 1999).

In the wake region of $z / H<0.75$, the maximum values of $k / u_{H}^{2}$ are observed at the position of $x / H=2.25$ under different stability conditions and are 0.14 for the stable condition, 0.13 for the neutral condition and 0.11 for the unstable condition, respectively. The maximum corresponds to the position where the velocity gradient is the steepest. Large wind shear appears near the recirculation region due to mechanical disturbance, which increases the TKE in the near-wake region of the hill and weakens the influence of thermal stratification on the TKE. The results are in agreement with those reported by Takahashi et al. (2005). However, the maximum values of $k / u_{H}^{2}$ are observed at the position of $x / H=1.125$ in the wind-tunnel test. This is because the RANS model overestimates the size of reverse flow region (Meroney et al. 1999). When atmospheric stratification is stable, the TKE is slightly higher than that under neutral and unstable conditions in the near-wake region of the hill. The effect of thermal stratification on $k / u_{H}^{2}$ gradually becomes dominant as the downwind distance increases (at $x / H=4.0$ and 5.5).

\section{c. Concentration field $K$}

Figure 10 shows the vertical profiles of normalized concentration $K$ at different horizontal positions in the simulation. At different downwind positions, in the range of $z / H<1.5$, the maximum concentration is observed under stable conditions and the minimum concentration is observed under unstable conditions. However, above the height of $z / H=1.5$, the concentrations for the unstable conditions are generally greater than those for the neutral and stable conditions. This indicates that the effect of the mechanical disturbance weakens with an increase in the height, and the pollutant concentration profiles are mainly dominated by thermal stratification over the height of $z / H=1.5$. In the wake region of the hill, the changes in concentration resulting from thermal stratification can be observed and the influence of thermal stratification on the pollutant dispersion becomes gradually obvious with the downwind distance increasing. The maximum concentrations for different conditions appear at the downwind position of $x / H=1.125$. The maximum values of $K$ are 13.5 for the stable condition at $z / H=1.15,0.58$ for the unstable condition at $z / H=$ 0.5 , and 2.0 for the neutral condition at $z / H=0.9$.
At the point of $x / H=1.125$, the ground concentrations are 0.35 for the stable conditions, 0.4 for the unstable conditions, and 0.5 for the neutral conditions. The concentrations for the stable conditions are slightly smaller than those for the unstable and neutral conditions.

The vertical profile of concentrations on the center plane $(y=0)$ of the hill is shown in Fig. 11. When atmospheric stratification is stable, the spreading distance of the plume is larger in the downwind direction because the vertical spread decreases. A region of higher concentration appears on the windward side of the hill. When atmospheric stratification is neutral, the plume rises, and the vertical spread of the plume is smaller than that under unstable conditions. The large high-concentration regions are observed on the windward side of the hill because of the impingement effect under stable conditions. The maximum normalized concentration $K=30.0$ on the windward side. When atmospheric stratification is unstable, the regions of higher concentration are small on the windward side and the maximum value of $K$ is 5.0. The highconcentration region appears between the source and windward side under neutral and unstable conditions; however, it can extend to the leeward side of the hill under stable conditions.

Figure 12 shows the horizontal profiles of pollutant concentration (at $z=0.01 \mathrm{~m}$ ) in the downwind direction of the hill. When atmospheric stratification is stable, the lateral spread of plume is smaller than that under neutral and unstable conditions. The horizontal spread of the plume under unstable conditions is slightly larger than that under neutral and stable conditions. This is because the intense turbulence mixing under unstable conditions increases the distribution range of the plume.

The maximum ground concentration $K=0.3$ for the unstable conditions is observed on the leeward side $(x / H=2.0)$. This is because the vertical spread of plume is intense due to strong turbulence mixing under unstable stratification. The maximum ground concentration appears at a distance far downwind $(x / H=8.5)$ and the value is still 0.3 . This is because stable stratification increases the horizontal transport and suppresses vertical motion of airflow. Furthermore, when $\operatorname{Fr}>1$, the flow structure under slightly stable conditions is similar to that under neutral conditions. Even through $\mathrm{Fr} \approx 10$, stable stratification still has a strong effect on flow and dispersion at a distance far downwind (Hunt and Snyder 1980), which is in agreement with their conclusions. The maximum ground concentration under neutral conditions $K=0.5$, which appears at the position of $x / H=4.0$. 

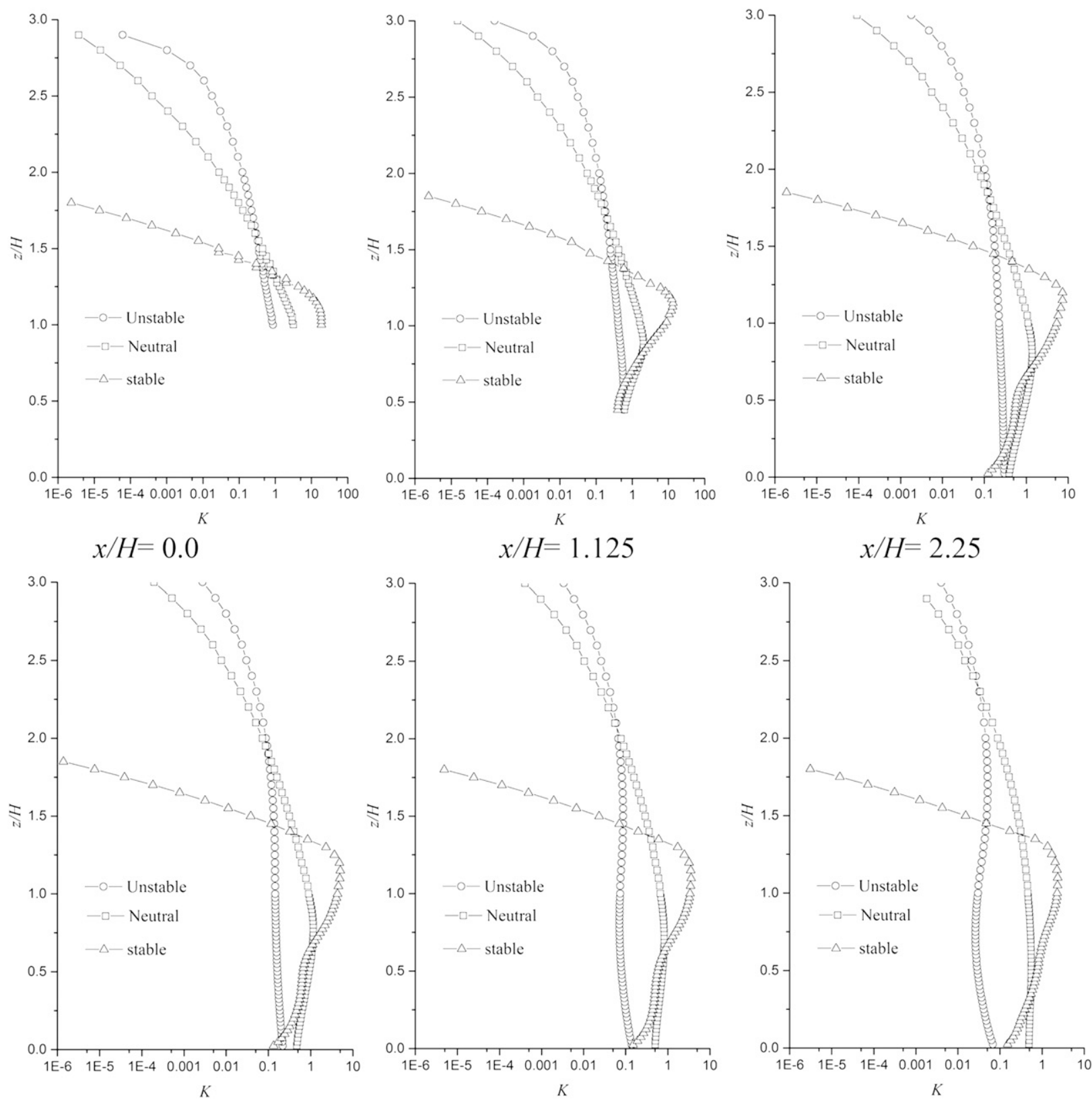

$x / H=3.0$

$x / H=4.0$

$x / H=5.5$

FIG. 10. Vertical profiles of mean concentration, $K$.

Overall, when atmospheric stratification is stable, the vertical spread of the plume is smaller than that under neutral and unstable conditions, and the downwind distance at where the maximum concentration appears is greater than that under neutral and unstable conditions. This is because stable stratification suppresses the vertical spread of the plume and increases the pollutant concentration in the wake region of the hill. When atmospheric stratification is unstable, the horizontal distribution of the plume is larger than that under neutral and stable conditions because intense turbulence mixing increases the dilution of the plume.

\section{Conclusions}

The simulation results for the effect of thermal stratification on the flow and dispersion around the hill show the following: 


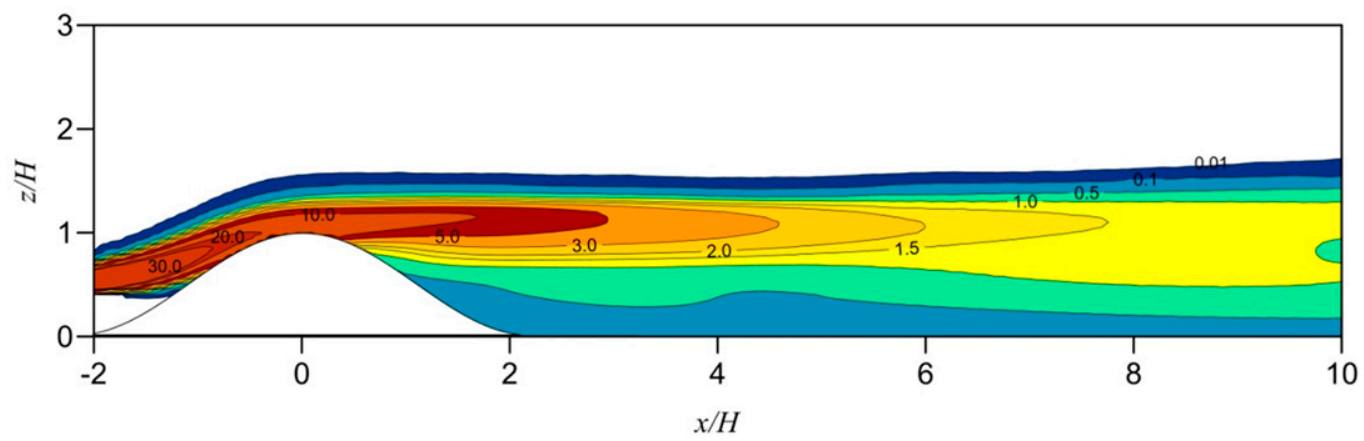

(a) Stable conditions

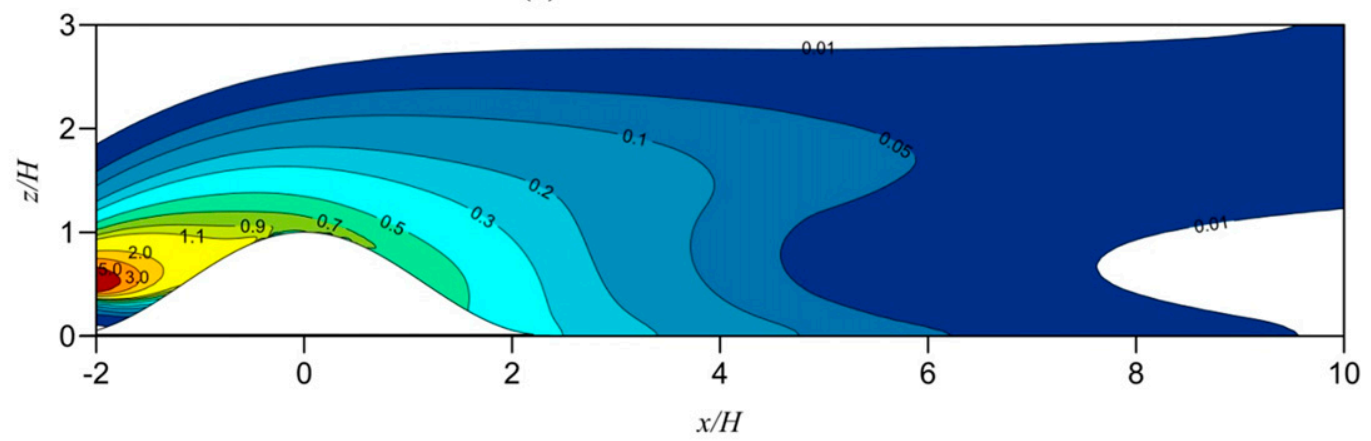

(b) Unstable conditions

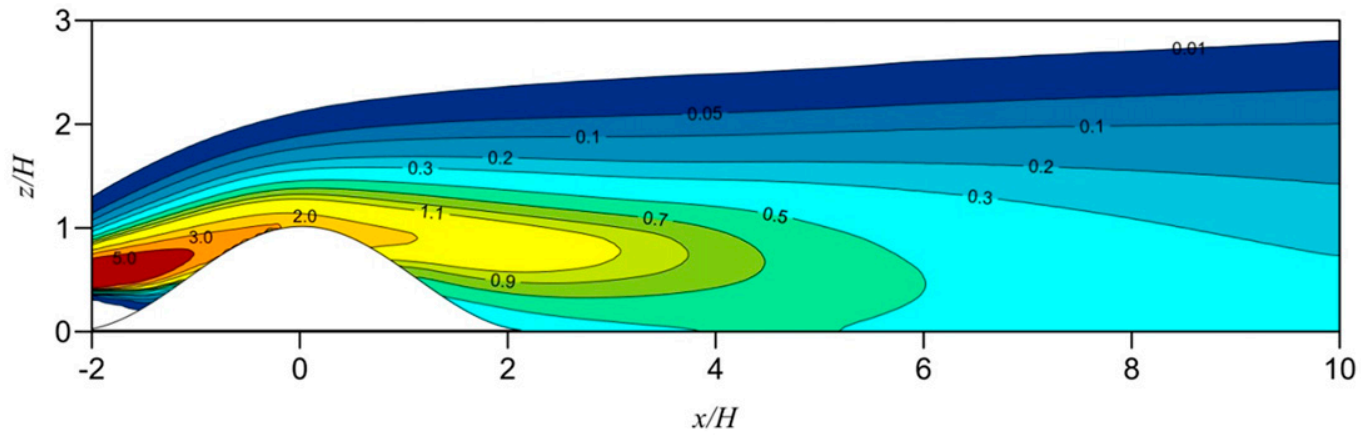

(c) Neutral conditions

FIG. 11. The vertical profile of concentrations on the center plane of the hill, $K$.

1) The greatest gradient of wind velocity appears at the hilltop, and the wind velocities are small in the wake region under different conditions. When atmospheric stratification is stable, the wind velocity decreases significantly in the near-wake region (including the recirculation region), and the velocity deficit is greater than that under neutral and unstable conditions. The influence of thermal stratification on velocity is not significant over the hilltop $(x / H=0.0)$ because of the dominant effect of mechanical disturbance. In the wake region, $u / u_{H}$ is large under neutral and stable conditions but relatively smaller under unstable conditions. When atmospheric stratification is stable, the velocity deficit in the range of $z / H<1.0$ is less than that under neutral and unstable conditions. The effect of thermal stratification on the flow field is inconspicuous under neutral and unstable conditions because the influence of mechanical disturbance produced by a hill is dominant. However, this effect under stable conditions is slightly conspicuous.

2) When atmospheric stratification is stable, the TKE is slightly less than that under neutral and unstable conditions, and the effect of thermal stratification on the turbulence intensity becomes gradually conspicuous with the distance increasing $(x / H=$ 4.0 and 5.5). The limitations of the RANS model cause a large discrepancy between the simulated results and wind-tunnel experiment data in the recirculation region. 


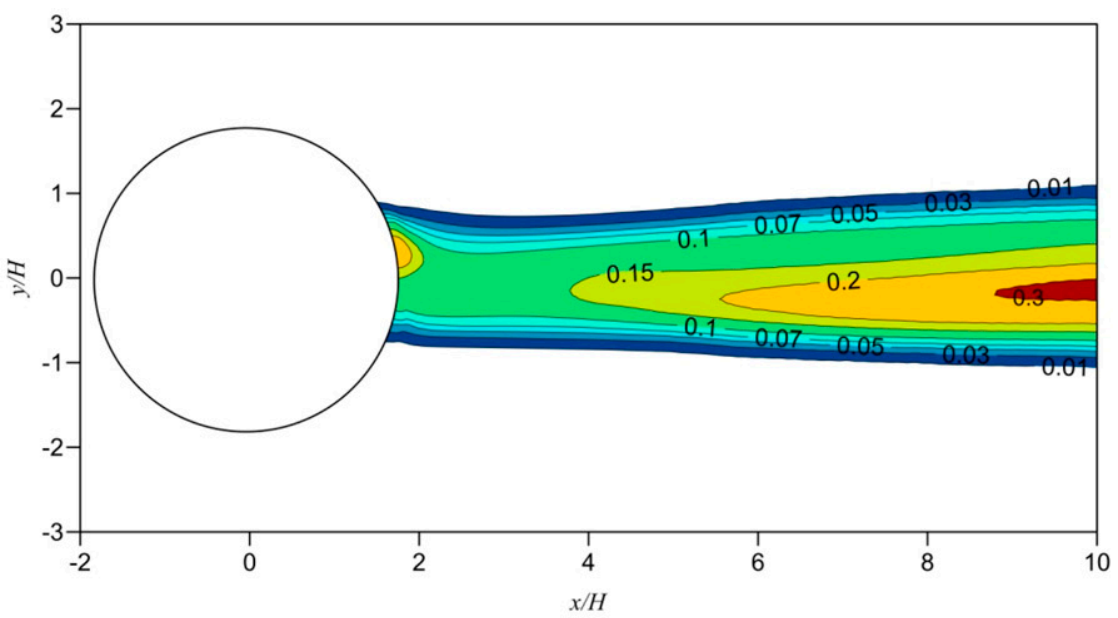

(a)Stable conditions

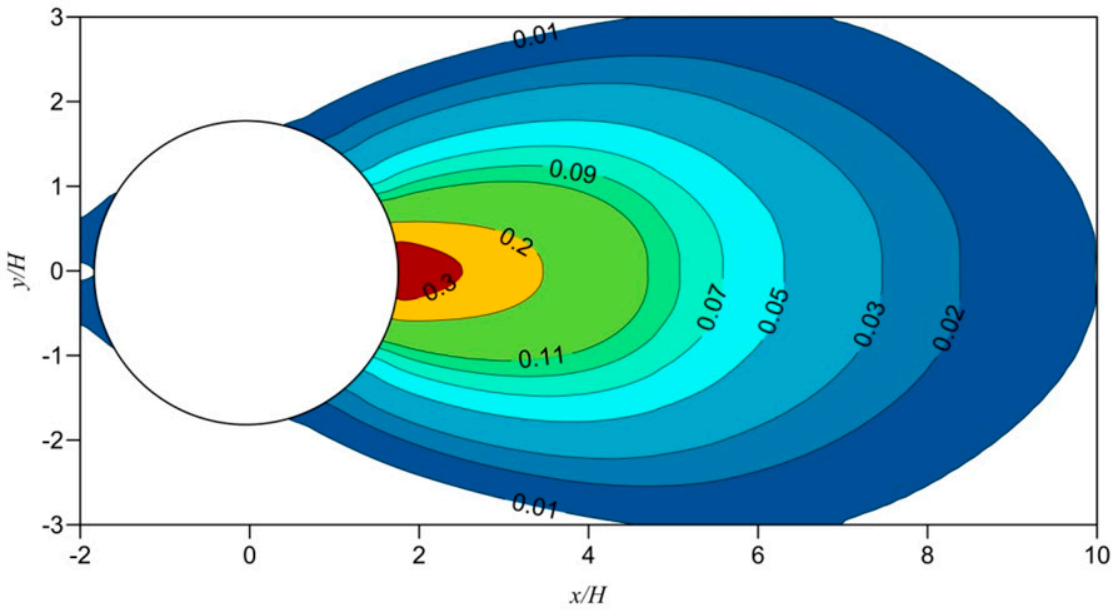

(b) Unstable conditions

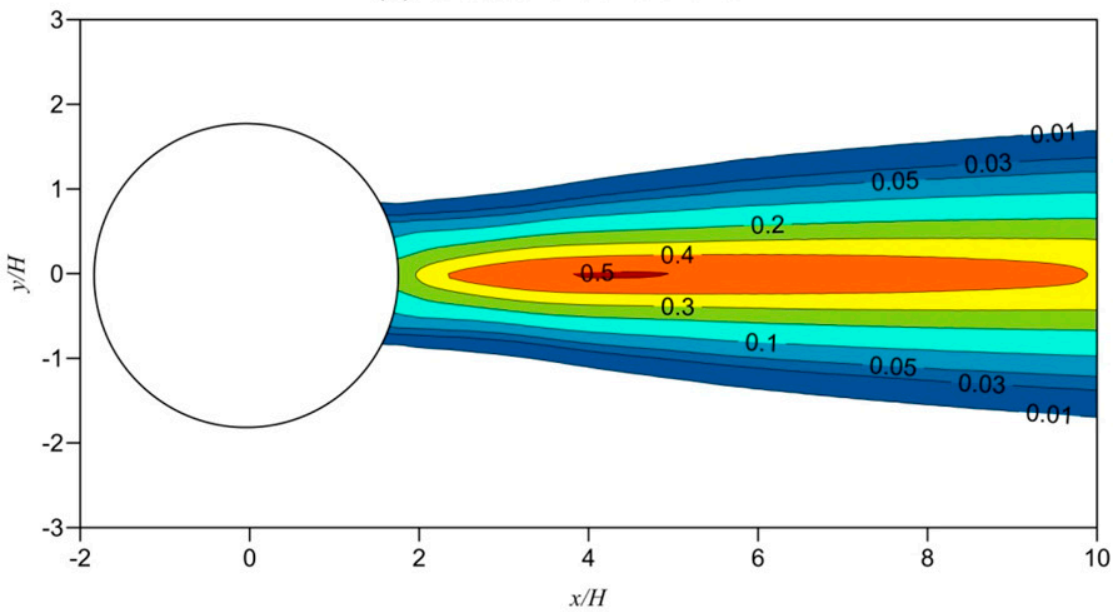

(c) Neutral conditions

FIG. 12. The horizontal profiles of pollutant concentration (at $z=0.01 \mathrm{~m}$ ) in the downwind direction of the hill, $K$. 
3) The maximum concentration at different downwind positions of hill appears at $x / H=1.125$ under different conditions. This is because greater TKE is produced by the mechanical disturbance near the wake region. Stable stratification increases the pollutant concentrations in the wake region. The vertical and horizontal distributions of concentration contours under unstable conditions are larger than those under neutral and stable conditions.

4) When atmospheric stratification is stable $(\mathrm{Fr} \approx 11)$, the large regions of higher concentration are observed on the windward side of the hill and the plume can reach a distance far downwind $(x / H=3.0)$. The high-concentration region on the windward side is small under unstable condition because of the intense turbulent mixing. The size of the regions under neutral conditions is smaller than that under stable conditions but larger than that under unstable conditions.

In conclusion, as an effective method, CFD simulation is able to evaluate the effect of thermal stratification and aerodynamic disturbance caused by obstacles (hills and buildings) on dispersion and flow. Numerical simulation is a suitable approach to address the near-field dispersion around a complex obstacle.

\section{REFERENCES}

Carruthers, D. J., and J. C. R. Hunt, 1990: Fluid mechanics of airflow over hills: Turbulence, fluxes, and waves in the boundary layer. Atmospheric Processes over Complex Terrain, Meteor. Monogr., No. 45, Amer. Meteor. Soc., 83-103.

Chang, C. Y., J. Schmidt, M. Dorenkamper, and B. Stoevesandt, 2018: A consistent steady state CFD simulation method for stratified atmospheric boundary layer flows. J. Wind Eng. Ind. Aerodyn., 172, 55-67, https://doi.org/10.1016/j.jweia.2017.10.003.

Cheng, W. C., C. H. Liu, and D. Y. C. Leung, 2009: On the correlation of air and pollutant exchange for street canyons in combined wind-buoyancy-driven flow. J. Atmos. Environ., 43, 3682-3690, https://doi.org/10.1016/j.atmosenv.2009.04.054.

Finnigan, J. J., 1988: Air flow over complex terrain. Flow and Transport in the Natural Environment: Advances and Applications, W. L. Steffen and O. T. Denmead, Eds., Springer, 183-229.

Hunt, J. C. R., and W. H. Snyder, 1980: Experiments on stably and neutrally stratified flow over a model three-dimensional hill. J. Fluid Mech., 98, 871-704, https://doi.org/10.1017/ S0022112080002303.

Kanda, I., and Y. Yamao, 2016: Passive scalar diffusion in and above urban-like roughness under weakly stable and unstable thermal stratification conditions. J. Wind Eng. Ind. Aerodyn., 148, 18-33, https://doi.org/10.1016/j.jweia.2015.11.002.

Kikumoto, H., R. Ooka, and K. Uehara, 2009: Large-eddy simulation of gaseous diffusion in street canyon with thermal stratification. Seventh Asia-Pacific Conf. on Wind Engineering, Taipei, Taiwan, International Association for Wind Engineering, 963-964, http://www.iawe.org/Proceedings/ 7APCWE/T1B_2.pdf.
Leo, L. S., M. Y. Thompson, S. Di Sabatino, and H. J. S. Fernando, 2016: Stratified flow past a hill: Dividing streamline concept revisited. Bound.-Layer Meteor., 159, 611-634, https://doi.org/ 10.1007/s10546-015-0101-1.

Meroney, R. N., B. M. Leitl, St. Rafailidis, and M. Schatzmann, 1999: Wind-tunnel and numerical modeling of flow and dispersion about several building shapes. J. Wind Eng. Ind. Aerodyn., 81, 333-345, https://doi.org/10.1016/S0167-6105(99) 00028-8.

Michalcová, V., Lausová, L., Skotnicová, I. and Pospíšil, S. 2016: Numerical and experimental models of the thermally stratified boundary layer. Trans. VSB-Tech. Univ. Ostrava Civ. Eng. Ser., 16 (2), 135-140, https://doi.org/10.1515/TVSB-2016-0024.

Murakami, S., 1993: Comparison of various turbulence models applied to a bluff body. J. Wind Eng. Ind. Aerodyn., 46-47, 2136, https://doi.org/10.1016/0167-6105(93)90112-2.

Okaze, T., and A. Mochida, 2017: Large-eddy simulation of nonisothermal flow around a building using artificially generated inflow turbulent fluctuations of wind velocity and air temperature. J. Heat Isl. Inst. Int., 12 (2), 29-34.

Olvera, H. A., and A. R. Choudhuri, 2006: Numerical simulation of hydrogen dispersion in the vicinity of a cubical building in stable stratified atmospheres. Int. J. Hydrogen Energy, 31, 2356-2369, https://doi.org/10.1016/j.ijhydene.2006.02.022.

Panofsky, H. A., and J. A. Dutton, 1984: Atmospheric Turbulence: Models and Methods for Engineering Applications. Wiley Interscience, $397 \mathrm{pp}$.

Patankar, S. V., and D. B. Spalding, 1972: A calculation procedure for heat, mass and momentum transfer in three-dimensional parabolic flows. Int. J. Heat Mass Transfer, 15, 1787-1806, https://doi.org/10.1016/0017-9310(72)90054-3.

Pontiggia, M., M. Derudi, V. Busini, and R. Rota, 2009: Hazardous gas dispersion: A CFD model accounting for atmospheric stability classes. J. Hazard. Mater., 171, 739-747, https:// doi.org/10.1016/j.jhazmat.2009.06.064.

Santos, J. M., N. C. Reis Jr., E. V. Goulart, and I. Mavroidis, 2009: Numerical simulation of flow and dispersion around an isolated cubical building: The effect of the atmospheric stratification. Atmos. Environ., 43, 5484-5492, https://doi.org/10.1016/ j.atmosenv.2009.07.020.

Snyder, W. H., 1981: Guidelines for fluid modeling of atmospheric diffusion. EPA Rep. EPA-600/8-81-0009, 185 pp., https://nepis.epa.gov/Exe/ZyPDF.cgi/2000BDW8.PDF?Dockey= 2000BDW8.PDF.

Takahashi, T., S. Kato, S. Murakami, R. Ooka, M. F. Yassin, and R. Kono, 2005: Wind tunnel tests of effects of atmospheric stability on turbulent flow over a three-dimensional hill. J. Wind Eng. Ind. Aerodyn., 93, 155-169, https://doi.org/ 10.1016/j.jweia.2004.11.003.

Tan, Z., J. Dong, Y. Xiao, and J. Tu, 2015: A numerical study of diurnally varying surface temperature on flow patterns and pollutant dispersion in street canyons. Atmos. Environ., 104, 217-227, https://doi.org/10.1016/j.atmosenv.2015.01.027.

Tominaga, Y., and T. Stathopoulos, 2017: Steady and unsteady RANS simulations of pollutant dispersion around isolated cubical buildings: Effect of large-scale fluctuations on the concentration field. J. Wind Eng. Ind. Aerodyn., 165, 23-33, https://doi.org/10.1016/j.jweia.2017.02.001.

- and —, 2018: CFD simulations of near-field pollutant dispersion with different plume buoyancies. Build. Environ., 131, 128-139, https://doi.org/10.1016/j.buildenv.2018.01.008. , A. Mochida, S. Murakami, and S. Sawaki, 2008: Comparison of various revised k-epsilon models and LES applied to flow 
around a high-rise building model with 1:1:2 shape placed within the surface boundary layer. J. Wind Eng. Ind. Aerodyn., 96, 389-411, https://doi.org/10.1016/j.jweia.2008.01.004.

van der Laan, M. P., M. C. Kelly, and N. N. Sørensen, 2016: A new k-epsilon model consistent with Monin-Obukhov similarity theory. Wind Energy, 20, 479-489, https://doi.org/10.1002/WE.2017.

Wood, N., 1995: The onset of separation in neutral, turbulent flow over hills. Bound.-Layer Meteor., 76, 137-164, https://doi.org/ 10.1007/BF00710894.

Yakhot, V., S. A. Orszag, S. Thangam, T. B. Gatski, and C. G. Speziale, 1992: Development of turbulence models for shear flows by a double expansion technique. Phys. Fluids, $\mathbf{4 A}$, 1510-1520, https://doi.org/10.1063/1.858424.

Yassin, M. F., 2013: A wind tunnel study on the effect of thermal stability on flow and dispersion of rooftop stack emissions in the near wake of a building. Atmos. Environ., 65, 89-100, https://doi.org/10.1016/j.atmosenv.2012.10.013.

- - S. Kato, R. Ooka, T. Takahashi, and R. Kouno, 2005: Field and wind-tunnel study of pollutant dispersion in a built-up area under various meteorological conditions. J. Wind Eng. Ind. Aerodyn., 93, 361-382, https://doi.org/ 10.1016/j.jweia.2005.02.005. 